\title{
More genomic resources for less-studied crops
}

\author{
Rajeev K. Varshney ${ }^{1,2,3}$, Jean-Christophe Glaszmann ${ }^{2,4}$, Hei Leung ${ }^{5}$ and \\ Jean-Marcel Ribaut ${ }^{2}$
}

\author{
${ }^{1}$ International Crops Research Institute for the Semi-Arid Tropics (ICRISAT), Patancheru 502324, A.P., India \\ ${ }^{2}$ Generation Challenge Programme (GCP), c/o CIMMYT, Int APDO Postal 6-641, 06600 Mexico, DF, Mexico \\ ${ }^{3}$ School of Plant Biology (M084), Faculty of Natural and Agricultural Sciences, The University of Western Australia (UWA), \\ 35 Stirling Highway, Crawley, WA 6009, Australia \\ ${ }^{4}$ UMR DAP, CIRAD, TA A96/03, Avenue Agropolis, 34398 Montpellier, Cedex 5, France \\ ${ }^{5}$ International Rice Research Institute (IRRI), DAPO Box 7777, Metro Manila 1301, The Philippines
}

\begin{abstract}
Many of the crop species considered to be minor on a global scale, yet are important locally for food security in the developing world, have remained less-studied crops. Recent years have witnessed the development of largescale genomic and genetic resources, including simple sequence repeat, single nucleotide polymorphism and diversity array technology markers, expressed sequence tags or transcript reads, bacterial artificial chromosome libraries, genetic and physical maps, and genetic stocks with rich genetic diversity, such as core reference sets and introgression lines in these crops. These resources have the potential to accelerate gene discovery and initiate molecular breeding in these crops, thereby enhancing crop productivity to ensure food security in developing countries.
\end{abstract}

\section{Introduction}

Trends in both the growth of the human population and its pattern of consumption in developing countries imply that the global demand for food will continue to grow for at least another 40 years. The focus now is not only on increasing the food supply, but also on improving its quality, particularly with respect to nutritional value [1,2]. Molecular breeding, which combines genotypic and phenotypic information, has emerged as a powerful approach and offers new perspectives to help address these challenges [3,4]. Genetic studies and molecular breeding approaches require basic genomic resources, such as molecular markers, genetic maps and sequence information, which were not available for several less-studied crop species until recently. Depending on the availability of those genomic resources, the main food crops of the world that are relevant to developing countries and are mandate crops of Consultative Group on International Agricultural Research (CGIAR), could be grouped into three tiers (Table 1). Considering the importance of less-studied crops in tiers 2 and 3 for balanced diet or income in developing countries, several international initiatives have contributed significantly to the development of genomic resources in these crops. The objective is twofold: first, to better understand the genetic nature and composition of their genome; and second, to afford molecular breeding

\begin{abstract}
Glossary
Chromosome segment substitution line (CSSL): Each CSSL carries a single or a few defined chromosome segments from a donor line in the genetic background of another line. These lines constitute useful resources for genetic studies, including detection and fine mapping of QTLs for the target traits. In particular, CSSLs can be used for the detection of QTLs with small additive effects that are masked by QTLs with large effects in filial generation $2\left(F_{2}\right)$ and recombinant inbred line populations.

Core collection: Generally contains $5-10 \%$ of the germplasm collection, and ideally represents at least $70 \%$ of the alleles in the whole collection.

Core reference set: A set of genetic stocks that are representative of the genetic resources of the crop and are used by the scientific community as a reference for an integrated characterization of its biological diversity.

Diversity array technology (DArT) markers: Represents a low-cost, highthroughput, robust marker system with minimal DNA requirements, which is capable of providing comprehensive genome coverage even for species that do not possess any genomic information. DArT is a segment of genomic DNA, the presence of which is polymorphic in a defined genomic representation. Expressed sequence tag (EST): Short $(300-500 \mathrm{bp})$, single-pass sequence obtained from the $5^{\prime}$ or $3^{\prime}$ end of mRNA (cDNAs). ESTs represent a snapshot of genes that are expressed at a given developmental stage or in a given tissue. Gene space: Long, gene-rich regions that contain the vast majority of genes, interrupted by long, gene-poor regions in a genome of a given species. Presence of gene space is a common feature of plants, including cereal species with large genome size owing to the abundance of repetitive DNA (retro and/or transposons) in their genome.

Introgression lines (ILs): A series of lines, each carrying a chromosomal segment from a donor (wild relative) genotype in the background of an elite genotype. Therefore, they constitute an "exotic library" where the entire wildspecies genome is partitioned among several lines, each carrying a single homozygous introgressed segment. The nearly isogenic nature of the ILs provides a relative advantage over other segregating populations when rapidly implementing a pyramiding approach via crosses and marker analysis. ILs constitutes an efficient source for the discovery and utilization of genes or QTLs that control traits of agricultural importance.

Map-based cloning (MBC): Also known as positional cloning, MBC is a universal strategy for cloning genes that are fine-mapped or genes whose genetic and physical positions are known.

Marker-assisted selection (MAS): A process of indirect selection for improving the traits of interest by employing markers, such as morphological, biochemical or DNA-based markers. DNA-based molecular markers have been considered the markers of choice for MAS in recent years.

Molecular breeding: A process of deployment of molecular markers in breeding practices for improving selection efficiency and for reducing the breeding cycles during cultivar improvement. MAS, marker-assisted recurrent selection, and genomic selection are different types of molecular breeding approaches.

Molecular marker: DNA-based genetic marker that can detect DNA polymorphisms both at the level of specific loci and at the whole-genome level. In recent years, a variety of molecular markers have become available. Restriction fragment length polymorphisms (RFLPs) were the first to be developed; others include random amplification of polymorphic DNAs (RAPDs), cleaved amplified polymorphic sequence (CAPS), SSRs and amplified fragment length polymorphisms (AFLPs). The most recently developed markers are SNPs and single-feature polymorphisms (SFPs).

Next generation sequencing (NGS) technologies: Modern sequencing technologies, driven by automation, that have surpassed Sanger sequencing
\end{abstract}

Corresponding author: Varshney, R.K. (r.k.varshney@cgiar.org). 
approach in throughput and in cost-effectiveness of sequencing. At present Roche-454/FLX, Illumina/Solexa and AB SOLiD are the NGS technologies that are in routine use for re-sequencing as well as de novo sequencing.

Tentative unique sequence (TUS): A non-redundant transcript sequence that is usually defined based on cluster analysis of short sequence reads generated by NGS technologies.

towards better performance of different cultivars in target environments. The Generation Challenge Programme (GCP), which links basic science with applied research through a broad network of plant scientists from diverse backgrounds, is one of the international initiatives in the 'research for development' arena (www.generationcp.org/ gcppartners.php?da=0646141) [5]. As a result of this effort, a significant amount of genomic resources as well as genetic stocks, such as core reference sets of CGIAR and national genebanks [6] and introgression libraries [7], have been developed in several less-studied crop species.

Studies conducted on plant models like Arabidopsis thaliana, Medicago truncatula and Lotus japonicus have opened the road to the development and application of new technologies and approaches [8]. The current challenge, however, is to deploy genomic information from model crops as well as crop-species-specific genomic and plantgenetic resources in breeding programmes. Here, we attempt to encapsulate progress achieved to date regarding the development of genomic resources and their applications for accessing genetic diversity and identifying favorable alleles for plant breeding. Challenges and opportunities for the deployment of these genomic and genetic resources in breeding programmes, particularly in developing countries, are also discussed.

Table 1. Availability of public domain genomic resources for each of the CGIAR/GCP mandate crops

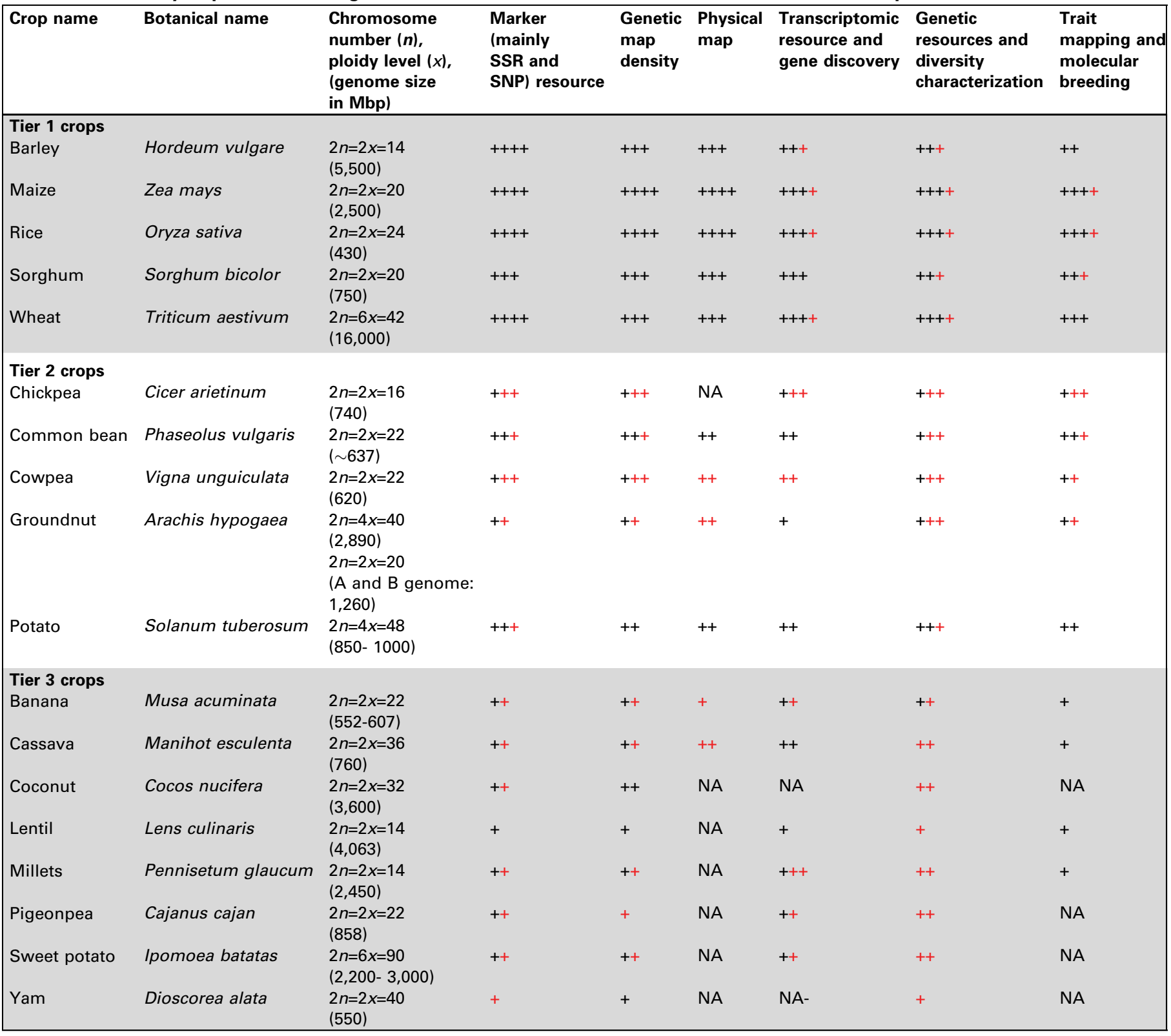

Abbreviations: NA, not available.

Availability of resources has shown by '+' sign as follows: +, basic; ++, moderate; +++, good; ++++, excellent. ' + ' colored red represents the contribution of GCP; those in black were already developed and are publicly available. 


\section{Current molecular marker technologies}

Molecular markers are key tools for assaying genetic variation. Advances in molecular genetic technology have generated a range of marker types, with many reliant on PCR. Some of these widely used marker systems include simple sequence repeats (SSRs), single nucleotide polymorphisms (SNPs) and diversity array technologies (DArTs) [9]. SSRs, or microsatellite markers, for various practical reasons, have been the leading marker types for breeding applications [10]. The DArT marker system has proven extremely effective in exposing genetic diversity in germplasm collections, and is well-suited for background selection in molecular breeding programmes [11]. Finally, SNPs, which have been the prime marker platform in human and animal genetics for some time, are now rapidly gaining momentum and are overtaking SSRs as the marker type of choice in plants as well, mainly because of their amenability for high-throughput genotyping and costeffectiveness [12,13].

\section{SSR and DArT markers}

Tier 1 species have benefited from an adequate supply of molecular markers, particularly SSRs (Table 1) [14]. A significant effort has therefore been dedicated recently towards developing SSRs in the tier 2 and 3 crops. Two major approaches have been deployed: the first is based on the construction of SSR-enriched genomic DNA libraries, and the second is centered on an in silico analysis of end sequences of clones from bacterial artificial chromosome (BAC) libraries or expressed sequence tag (EST) sequences. As a result, hundreds of SSR markers have become available in several crops, such as chickpea [15,16], common bean $[16,17]$, groundnut $[16,18,19]$ and pigeonpea $[16,20]$ (Table 1). However, the cost of developing SSR markers, especially via the enriched library route, remains rather high.

The need for a whole genome assay, required both for the characterization of diversity and for development of genetic maps, has led to the application of DArT technology to several tier 2 and 3 crop species (www.diversityarrays. com/genotypingserv.html). Although the first set of DArT arrays were developed for pearl millet, potato, sweet potato, cassava, yam, coconut and Musa, the expansion of existing DArT arrays was accomplished for chickpea, groundnut and pigeonpea (Table 2). DArT markers have since proven useful in the areas of germplasm collection diversity assessment [21,22], genome mapping [22-24] and gene tagging [25], and should be useful in marker-assisted selection (MAS), especially for monitoring the recovery of the recurrent parent genome.

\section{BAC libraries}

BAC libraries are an important component of physical mapping, map-based cloning, and the analysis of gene and genome structure and function. BAC libraries that provide extensive genome coverage have been established in rice [26], maize [27], wheat [28], barley [29], sorghum [30], soybean [31], common bean [32] and potato [33], among others. Recently, BAC libraries have been constructed in a number of less high profile crop species, such as cowpea, cassava and groundnut [16].

Two independent cowpea BAC libraries, comprising $\sim 74000$ clones and $17 \times$ genome coverage, have been developed and high-information-content fingerprinting (HICF) analysis [34] of 60000 of these clones has allowed their assembly into 790 contigs ([35], http://phymap. ucdavis.edu/cowpea/). A similar project in cassava has produced a BAC library of 72000 clones that represents approximately $10 \times$ coverage of the genome. Following the HICF method, 58 000 clones have been assembled into 2104 contigs ([36], http://cassava.igs.umaryland.edu/). Although a $6.5 \times$ groundnut library had already been established [37], two new BAC libraries, one derived from the groundnut A genome progenitor Arachis duranensis, and the other from its B genome progenitor, Arachis ipaënsis, have been constructed recently [38] for finger-

Table 2. CGIAR/GCP crop species for which DArT markers are available

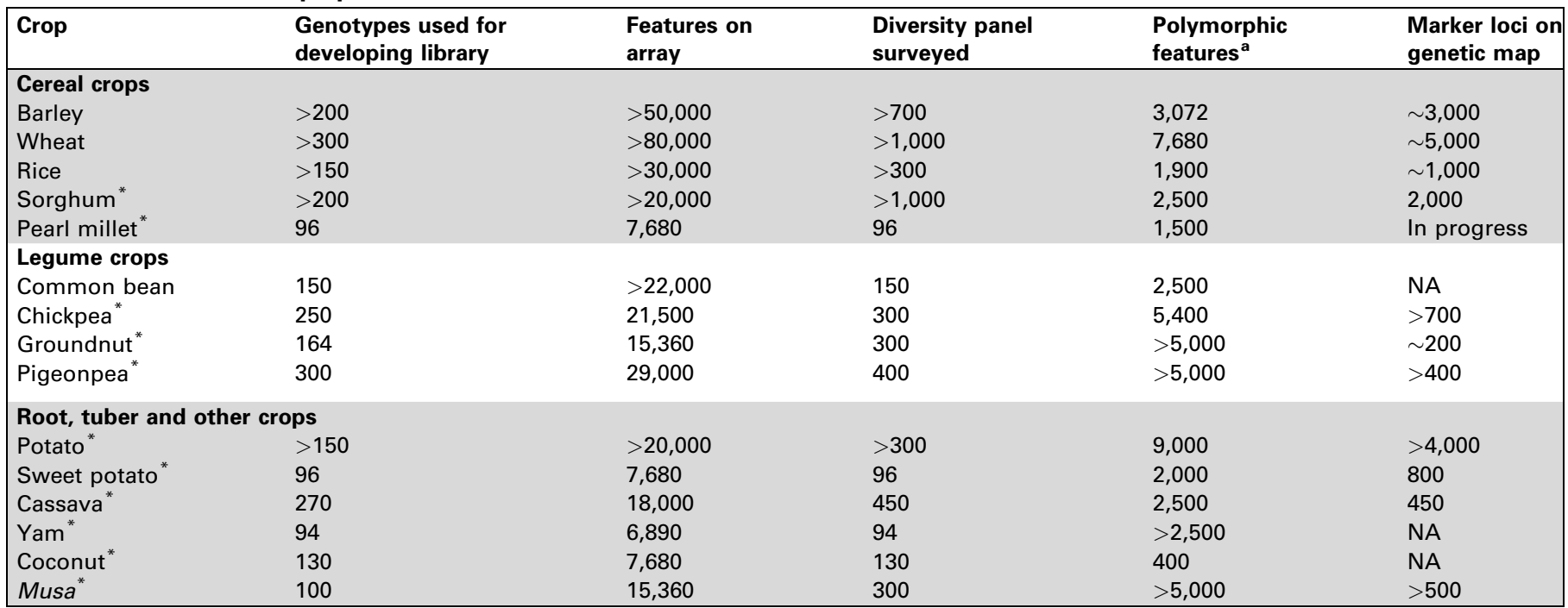

Abbreviation: NA, not available.

Source: DArT Pty Ltd., Australia (www.diversityarrays.com).

"Species for which the research was supported by GCP [65]. A complete list of crop species for which DArT arrays are available is posted at www.diversityarrays.com.

${ }^{a}$ The number of informative markers in chickpea, groundnut and pigeonpea includes related wild species. The number for yam is a projection of current work. 
printing. In summary, the physical maps of several tropical crop species are now under development. As physical maps are integrated with genetic maps, they will provide the framework for full genome sequencing.

\section{Sequence-based, next generation genomic resources}

Recent years have witnessed the advent of a number of high-throughput sequencing [39-41] as well as genotyping technologies $[12,13]$. These approaches have great potential to facilitate development of genomic resources even in those crops that have a shortage of genomic resources. Such potential and applications of next generation sequencing (NGS) technologies like Roche/454 and Illumina/Solexa sequencing have been discussed in detail elsewhere [39-41]. In fact, as a result of the use of NGS technologies, whole-genome sequences are becoming available in these plant species, which did not have even basic genomic resources like marker repertoire and genetic maps [42]. Therefore, these approaches have been used for developing sequencebased genomic resources at a fast pace in some tier 2 and 3 crop species, which were considered 'orphan crops' in the past (Table 1) [16].

\section{Transcriptomic resources}

Several temperate cereal ESTs have been in the public domain for some time (dbEST release 042310, www.ncbi.nlm.nih.gov/dbEST/), but this has not been the case for less-studied crops like cowpea, chickpea, pearl millet or sweet potato. In recent years, however, because of concerted efforts of GCP partners and others, transcriptomic resources in the form of ESTs (generated by Sanger sequencing) and/or short transcript reads (STRs; generated by NGS technologies) have been generated [16]. Large number of ESTs (e.g. 180000 for cowpea [43], 20 000 for chickpea [44] and 11000 for pigeonpea [45]) have become available recently. On the other hand, Roche/454 has been used to generate $>435000$ chickpea and $\sim 495000$ pigeonpea STRs $[16,39]$. Similarly, 113000 tentative unique sequences (TUSs) for pearl millet [46] and $\sim 60000$ TUSs for sweet potato [47] have been defined based on STRs.

\section{SNP resources and high-throughput genotyping platforms}

Technological advances in both detection and robotics have seen SNPs largely replacing SSRs as markers for mammalian genetics, and their use in plants is expanding [13]. International collaborations have generated large-scale SNP resources and genotyping platforms in rice (www. ricesnp.org) [48], maize (www.maizegenetics.net) [49,50], wheat (http://wheat.pw.usda.gov/SNP/new/index.shtml) [51], barley [52] and soybean [53]. Initiatives like GCP have extended SNP technology to various tropical crop species. Two main approaches have been applied for SNP discovery: the first is based on available EST and STR sequences, and the second on allele re-sequencing of candidate genes or BAC ends. The cowpea SNP programme has delivered $\sim 10000 \mathrm{SNPs}$ from an in silico comparison of $\sim 183000$ ESTs generated from $>10$ cultivars (http://harvest.ucr.edu/) [43]. Re-sequencing of an average of $\sim 600$ genes per species among the mapping parents used in cowpea, chickpea, common bean, pigeonpea, and one of the diploid progenitors of groundnut has identified $\sim 16000$ SNPs in these tropical legume species $[16,54]$. In the cases of chickpea and pigeonpea, Illumina/ Solexa sequencing has also been used to generate STRs for parental genotypes of mapping populations. Alignment of STRs with respective transcriptome assemblies (TUSs) has allowed identification of several thousands SNPs in each chickpea and pigeonpea at the International Crops Research Institute for the Semi-Arid Tropics (ICRISAT: www.icrisat.org) [16].

A number of high-throughput SNP genotyping platforms is currently available [13]; among these, the GoldenGate assay has become popular because its unit data point cost is very competitive. A 1536-feature GoldenGate assay has been developed for cowpea [43], as well as a 768feature GoldenGate assay for cowpea, chickpea, piegonpea, common bean and one of the diploid progenitors of groundnut $[16,54]$. Similarly, a SNP-based GoldenGate assay has been developed for cassava. The advent of SNP genotyping has led to the shift from the low-throughput primarily manual world of SSRs to high-throughput platforms that are powered by robotics and automated scoring. As a result, genotyping costs with SNPs have decreased by up to 10-fold, whereas data throughput has increased by the same magnitude, which has enabled a great leap in genotyping efficiency [55].

\section{Genome or gene space sequencing}

Rice was the first crop for which genome sequencing was completed [56-58], and genome sequences have recently become available for sorghum [59] and maize [60]. Similar efforts are underway for wheat [61] and barley [62]. Although NGS technologies were originally meant for resequencing, ever-increasing sequence read length and advances in bioinformatics have made NGS possible for undertaking de novo genome sequencing [41,42]. Although gene space was sequenced for cowpea [63], these novel approaches have enabled complete genome sequencing for species like cassava (www.phytozome.net/cassava) and potato (www.potatogenome.net), and some efforts have been initiated in pigeonpea (www.icrisat.org/gt-bt/IIPG/ Home.html). Sequencing of the AA genome of Musa has also been announced by Genoscope and the French Centre for International Agricultural Research (CIRAD) (www.musagenomics.org). In the coming years, sequences of either the whole genome or at least the gene space should become available for a majority of crop species, including the less-studied species.

\section{Novel and better-characterized sources of genetic diversity}

The use and creation of genetic diversity to stack favorable alleles into germplasm for enhanced agronomic performance in target environments is a primary task of the plant breeder. Worldwide, 1400 ex situ genebanks curate six million accessions, which cover most of the common crop species [6]. Direct identification of favorable alleles in genebank accessions, however, is often masked due to poor adaptation or poor agronomical performance of the genebank material. Therefore, several approaches have been 
Table 3. Development of core reference set for CGIAR mandate crop species

\begin{tabular}{|c|c|c|c|c|}
\hline Crop $^{a}$ & $\begin{array}{l}\text { Composite or core collection for } \\
\text { molecular characterization }\end{array}$ & SSR markers & $\begin{array}{l}\text { Size of reference set } \\
\text { defined }\end{array}$ & Lead institution and partners $^{b}$ \\
\hline \multicolumn{5}{|l|}{ Tier 1 crops } \\
\hline Barley & 2,676 & 14 & 300 & ICARDA, CAAS \\
\hline Rice & 2,757 & 50 & Analysis in progress & IRRI, CAAS, CIAT, WARDA, Agropolis \\
\hline Sorghum & 3,393 & 39 & 345 & ICRISAT, Agropolis, CAAS \\
\hline Wheat & 3,000 & 50 & 372 & CIMMYT, Agropolis-CAAS-ICARDA \\
\hline Common bean & 625 & 36 & 192 & CIAT, EMBRAPA \\
\hline Cowpea & 1,871 & 16 & 345 & IITA \\
\hline Groundnut & 991 & 21 & 300 & ICRISAT, EMBRAPA \\
\hline Potato & 1,000 & 50 & Analysis in progress & CIP \\
\hline \multicolumn{5}{|l|}{ Tier 3 crops } \\
\hline Banana & 327 & 48 & 96 & Bioversity, IITA, Agropolis \\
\hline Foxtail millet & 500 & 20 & 200 & ICRISAT \\
\hline Lentil & 1,000 & 24 & 150 & ICARDA \\
\hline Pearl millet & 1,000 & 20 & 300 & ICRISAT \\
\hline Pigeonpea & 1,000 & 20 & 300 & ICRISAT \\
\hline Sweet potato & 500 & 50 & Analysis in progress & CIP \\
\hline Yam & 350 & 20 & 342 & IITA \\
\hline
\end{tabular}

${ }^{a}$ Crop species in which molecular characterization has been supported by GCP.

bLead institute is listed first, and collaborating institutes follow: Agropolis: Agropolis International, France (www.agropolis.fr); Bioversity: Bioversity International (www.bioversityinternational.org); CAAS: Chinese Academy of Agricultural Sciences, China (www.caas.net.cn); CIAT: International Center for Tropical Agriculture, Colombia (www.ciat.cgiar.org); CIMMYT: International Maize and Wheat Improvement Center, Mexico (www.cimmyt.org); CIP: International Potato Center, Peru (www.cipotato.org); EMBRAPA: Brazilian Agricultural Research Corporation, Brazil (www.embrapa.br); ICARDA: International Center for Agricultural Research in the Dry Area, Syria (www. icarda.org); IITA: International Institute of Tropical Agriculture, Nigeria (www.iita.org); IRRI: International Rice Research Institute, Philippines (www.irri.org); WARDA: The Africa Rice Center, Benin (www.warda.org).

suggested to create, characterize and tap genetic diversity in the breeding programme; two cases in which significant efforts have been made for assessing and capturing favorable alleles for breeding are discussed in the following sections.

\section{Core reference sets}

Most likely, a very small portion of the ex situ accessions has been used in plant breeding programmes. With an objective to enhance use of germplasm in breeding, various formulations for selecting representative sub-collections have been derived from the original concept of the core collection [64]. For each crop listed in Table 3, a systematic molecular characterization of germplasm from various collections has been conducted to define the core reference set to enable rationale sub-sampling. The central idea is to promote the use of a common set of representative material by the scientific community. This concept of core reference set to help R.E.A.D. (represent existing diversity - enter the whole collection - assess phenotype variation - dissect trait-gene associations) germplasm has been described [6].

As a result of concerted efforts of scientists from CGIAR centres and national programmes coordinated and supported by GCP, genotyping of $\sim 32000$ accessions, which represents global composite collections of 21 species from 12 CGIAR/advanced research institute (ARI)/national agricultural research system (NARS) genebanks has been undertaken at 14-50 SSR loci or with DArT arrays (Table 3) [65]. The genotypic data were initially used to design a core reference set for each species, which consisted of 96359 accessions. The aim of the core reference set was to capture $\sim 80 \%$ of the molecular diversity present in the sampled germplasm [66]. The next step is to gather robust phenotypic data sets, assembled from trials conducted under a range of agro-climatic conditions and genome-wide high-density marker data on these core reference sets. These data, although challenging, are essential for the identification of phenotype/genotype associations that might deliver marker tags for agronomic traits. In addition, multi-location field trials could also succeed in identifying lines that might be included in breeding efforts because they perform well with respect to a specific trait of interest.

\section{Introgression libraries}

Depending on the presence of a superior allele for a trait, such as disease resistance, in a crop relative or wild species, the identification of favorable alleles can be complicated because of often-poor agronomic performance of such non-adaptive germplasm. In such cases, production of a complete set of chromosome segment substitution lines (CSSLs) [e.g. recombinant inbred introgression lines (ILs)] provides avenues to explore the largely untapped reservoir of useful alleles of interest that remain in the wild species. Such a case is shown in Figure 1, in which the ILs of rice have been developed by using chromosome segments of Oryza meridionalis. Such lines enable assessment of the phenotypic impact of the wild species genome in parts while keeping the genetic background of the cultivated recipient genotype. This strategy has been widely utilized for introgression of favourable alleles from wild relatives in tomato, rice, wheat and barley [4]. 


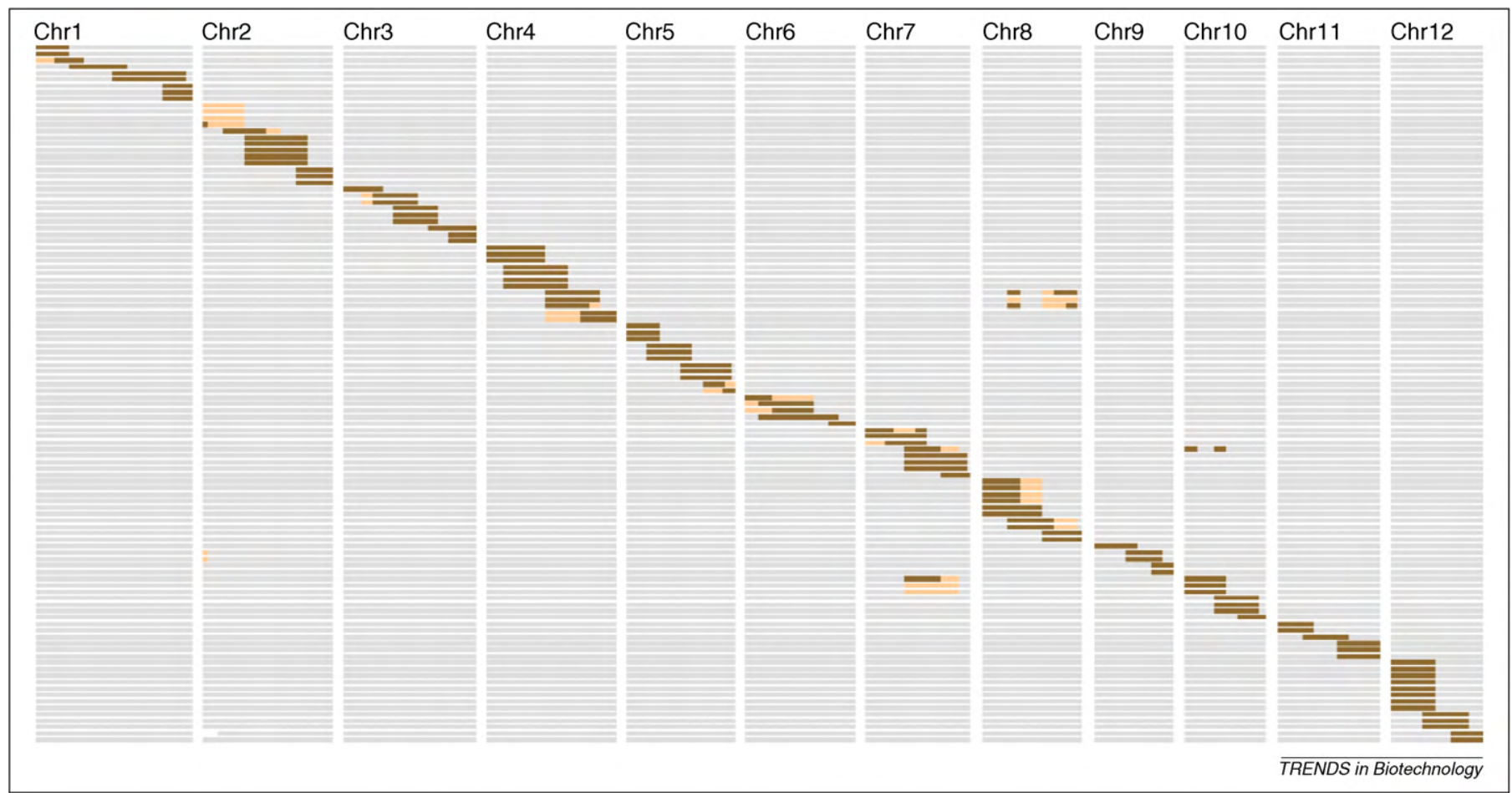

Figure 1. Graphical genotypes of a set of rice CSSLs, selected in the $\mathrm{BC}_{3}$ generation of a cross between cultivated rice and $O$. meridionalis by researchers at $\mathrm{CIAT}$ (Source: Mathias Lorieux and colleagues). Background selection was carried out using a genome-wide set of SSR and miniature inverted repeat transposable elements markers. The filled brown bars represent $O$. meridionalis segments, and the orange bars represent fragment reduction or background recovery. Abbreviation: Chr, chromosome.

Inspired by these success stories, the International Center for Tropical Agriculture (CIAT) in Colombia in collaboration with the Institut de Recherche pour le Développement (IRD), and CIRAD in collaboration with the Institut Sénégalais de Recherches Agricoles (ISRA) have developed ILs to transfer exotic alleles into elite lines of rice and groundnut, respectively. The donors of the various sets of Oryza spp. ILs involve Oryza glaberrima, O. meridionalis, O. rufipogon, O. glumaepatula and $O$. barthii, and the ILs have featured in a variety of genetic analyses and strategic breeding crosses. An example of the former is the use of ILs derived from the cross $O$. sativa with $O$. glaberrima to identify quantitative trait loci (QTLs) for plant height, tiller number, panicle length, male fertility, grain weight and grain yield. Of particular note is the genetic characterization and mapping of a major QTL that underlies resistance to the stripe necrosis virus [67]. Introgression in groundnut has been based on a cross between a cultivated type and synthetic tetraploid derived from a hybrid between the diploid progenitor species A. ipaënsis and $A$. duranensis [68]. The level of marker information between the mapping population parents has allowed for the construction of a coarse-scale genetic map (298 loci spread over 21 linkage groups, mean inter-marker distance of $6.1 \mathrm{cM}$ ). A back-cross strategy has been applied to the $F_{1}$ hybrid to reduce the proportion of the progenitor genome present, and these materials are seen as having substantial potential to extend the genetic base of cultivated groundnut.

\section{The impact of genomic resources on breeding}

The productivity of most tropical crops is limited by a combination of biotic and abiotic constraints. For most of these, plants have evolved variation for the level of tolerance or resistance. A crucial pre-requisite for the deployment of markers to support stress tolerance or resistance breeding is the development of a genetic map, followed by identification of gene-based or gene-linked markers to be used in a predictive mode for plant breeding, such as MAS [69]. However, until a few years ago, maps in cowpea, chickpea, pigeonpea, pearl millet and sweet potato were either non-existent or displayed an inadequate level of marker density. The far-reaching improvement in molecular marker technology witnessed over the past few years has helped to put in place serviceable genetic maps in all these species (Table 1). As an example, an AFLP-based cowpea map has recently been replaced by a 928-SNP marker map that consists of 11 linkage groups with a mean inter-marker distance of $<1 \mathrm{cM}$ [43]. The situation in groundnut is even more extreme: marker polymorphism in the species had been thought to be too poor to construct a genetic map, but now SSR-based linkage maps have been constructed for both diploid progenitors $[18,70]$ and tetraploid species [71]. The reference chickpea genetic map developed at ICRISAT comprises $>1500$ data points $[15,16]$, and mapping projects in pearl millet and sweet potato are being conducted at ICRISAT and the International Potato Center, Peru (CIP), respectively.

Based on the availability of genomic resources such as markers, genetic maps and transcriptomic resources, QTL identification or gene cloning projects have been approached in several less-studied crops for both biotic and abiotic stress (Table 1). These include aluminium toxicity tolerance in sorghum [72], virus resistance in cassava [73], and drought tolerance in cowpea, groundnut, chickpea and common bean (www.generationcp.org/gcptli/) [16]. While cloned genes (e.g. for aluminium tolerance in sorghum [72]) are being used for mining the superior 
alleles in African germplasm, the major QTLs identified for root traits in chickpea are being used in marker-assisted backcrossing (MABC) programmes in elite cultivars of India and Africa. By using the ICC 4958 genotype as a donor genotype, and JG 11 as a recurrent genotype, MABC has led to development of $\mathrm{BC}_{3} \mathrm{~F}_{3}$ lines at ICRISAT. After multiplication of seeds of these lines, agronomic performance will be evaluated in targeted environments. In the case of cassava, the MAS approach has already been successfully utilized to develop 14 genotypes that combine cassava mosaic disease (CMD) resistance and high yields by using eight markers associated with the dominant CMD2 gene for CMD [73]. Similarly, MAS efforts in pearl millet have led to the development of an "HHB 67 Improved" hybrid for downy mildew (DM)-resistance [74]; this study has demonstrated that, while conventional backcross transfer of DM resistance to improve parental lines took nearly nine years, a marker-based approach facilitated it in just three years.

\section{A bright future for genomics-assisted breeding in the developing world}

Most of the key developing country crops now find themselves in a situation in which genomic resources are sufficient to support meaningful genetic studies and molecular breeding [55]. Several successful genomics-assisted breeding programmes have been built through collaborations between CGIAR institutes and NARS partners [73-76]. Various bottlenecks still impede adoption in these countries; limited human resources, inadequate field infrastructure and limited capacity in information management remain major challenges. The magnitude of these challenges is exacerbated where it is important to breed for biotic (pests or diseases) and abiotic (drought, heat, cold or salinity) stresses, thus making accurate phenotyping challenging. On a positive note, the history of formal breeding in developing countries has been short, thus allowing for greater potential for improvement relative to genetic gains obtained in developed countries. However, through virtual platforms, such as the Integrated Breeding Platform (http://mbp. generationcp.org/) initiated in 2009 and coordinated by GCP in collaboration with the Bill \& Melinda Gates Foundation, further aided by the information and communication technology revolution, breeders in developing countries will soon have better access to genomic resources, advanced laboratory services, and robust analytical and data management tools. The task remains challenging as the Platform is intended to serve a broad range of users who are working on different crops for different environments [55].

Although few economic analyses have been conducted to assess the potential impacts of molecular breeding in developing countries, there are some well-known examples of success. One of them is the development of submergencetolerant rice cultivars through MABC, which has improved yields in $>15$ million hectares of rain-fed lowland rice in South and Southeast Asia [77]. A recent ex ante economic study of molecular breeding of rice for tolerance to salty and low-phosphorus soils in selected Asian countries has estimated that the method saves a minimum of 2-3 years, which results in significant incremental benefits in the range of $\$ 300-800$ million USD [78].
Large-scale genomic resources and specialized genetic stocks that have become available in tier 2 and 3 crops, which have been less-studied crops until recently, are expected to enhance molecular breeding such as MABC and marker-assisted recurrent selection (MARS) [55]. This will lead to enhanced crop productivity and, in turn, increased food security in developing countries. It is, however, unrealistic to project that large-scale molecular breeding activities will be conducted in the near-term in a broad set of developing countries, and the impact of this approach will be hardly homogeneous in its implementation [55]. The prospects are bright for breeders in some developing countries, particularly the newly industrialized ones with sustainable breeding programmes that benefit from institutional support. These countries should take advantage of large international initiatives by accessing germplasm data, genetic resources, genomic tools, and methodologies that will allow them to conduct efficient genetic studies and molecular breeding.

\section{Acknowledgements}

The authors are thankful to the entire GCP community for their hard work that led to the development of genomic resources and specialized genetic stocks mentioned in this article. Special thanks are due to Matthew Blair (CIAT, Columbia), Tim Close (University of CaliforniaRiverside, USA), Doug Cook (University of California-Davis, USA), Pooran Gaur (ICRISAT, India), Marc Ghislain (CIP, Peru), Tom Hash (ICRISAT, India), Sarah Hearne (International Institute of Tropical Agriculture, Nigeria), Andrzej Kilian (DArT Pty Ltd, Australia), JeanFrancois Rami (CIRAD, France), Mathias Lorieux (CIAT, Colombia), Andrew Paterson (University of Georgia, USA), Pablo Rabinowicz (University of Maryland, USA), Roland Schafleitner (CIP, Peru) and Hari Upadhyaya (ICRISAT, India) to share useful information presented in this review. Thanks are also due to Robert Koebner (smartEnglish, UK), and Abhishek Bohra, Anuja Dubey and Priyanka Puvvada from ICRISAT for their help while preparing and editing the MS.

\section{References}

1 Godfray, H.C.J. et al. (2010) Food security, The challenge of feeding 9 billion people. Science $327,812-818$

2 Alston, J.M. et al. (2009) Agricultural research, productivity, and food prices in the long run. Science 325, 1209-1210

3 Tester, M. and Langridge, P. (2010) Breeding technologies to increase crop production in a changing world. Science $327,818-822$

4 Varshney, R.K. and Dubey, A. (2009) Novel genomic tools and modern genetic and breeding approaches for crop improvement. J. Plant Biochem. Biotechnol. 18, 127-138

5 Ribaut, J-M. et al. (2008) International programs and the use of modern biotechnologies for crop improvement. In Genomics of Tropical Crop Plants (Moore, P. and Ming, R., eds), pp. 21-63, Springer

6 Glaszmann, J.C. et al. (2010) Accessing genetic diversity for crop improvement. Curr. Opin. Plant Biol. 13, 167-173

7 Zamir, D. (2001) Improving plant breeding with exotic genetic libraries. Nat. Rev. Genet. 2, 983-989

8 Koebner, R.M.D. and Varshney, R.K. (2006) Development and application of genomic models for large crop plant genomes. In Model Plants and Crop Improvement (Varshney, R.K. and Koebner, R.M.D., eds), pp. 1-9, CRC Press

9 Jones, N. et al. (2009) Markers and mapping revisited, finding your gene. New Phytol. 183, 935-966

10 Gupta, P.K. and Varshney, R.K. (2000) The development and use of microsatellite markers for genetics and plant breeding with emphasis on bread wheat. Euphytica 113, 163-185

11 Kilian, A. et al. (2005) The fast and the cheap, SNP and DArT-based whole genome profiling for crop improvement. In Proceedings of the International Congress: In the Wake of the Double Helix, from the Green Revolution to the Gene Revolution (Tuberosa, R., et al., eds.) pp. 443461, Avenue Media 
12 Gupta, P.K. et al. (2008) Array-based high-throughput DNA markers for crop improvement. Heredity 101, 5-18

13 Varshney, R.K. (2010) Gene based marker systems in plants, high throughput approaches for marker discovery and genotyping. In Molecular Techniques in Crop Improvement (Jain, S.M. and Brar, D.S., eds), pp. 119-140, Springer

14 Varshney, R.K. et al. (2006) Advances in cereal genomics and applications in crop breeding. Trends Biotechnol. 24, 490-499

15 Nayak, S.N. et al. (2010) Integration of novel SSR and gene-based SNP marker loci in the chickpea genetic map and establishment of new anchor points with Medicago truncatula genome. Theor. Appl. Genet. $120,1415-1441$

16 Varshney, R.K. et al. (2009) Orphan legume crops enter the genomics era! Curr. Opin. Plant Biol. 12, 202-210

17 Blair, M.W. et al. (2009) Development of microsatellite markers for common bean (Phaseolus vulgaris L.) based on screening of nonenriched, small-insert genomic libraries. Genome 52, 772-782

18 Moretzsohn, M.C. et al. (2005) A microsatellite-based, gene-rich linkage map for the AA genome of Arachis (Fabaceae). Theor. Appl. Genet. 111, 1060-1071

19 Cuc, L.M. et al. (2008) Isolation and characterization of novel microsatellite markers and their application for diversity assessment in cultivated groundnut (Arachis hypogaea). BMC Plant Biol. 8, 55

20 Varshney, R.K. et al. (2010) Pigeonpea genomics initiative (PGI), an international effort to improve crop productivity of pigeonpea (Cajanus cajan L.). Mol. Breed. DOI: 10.1007/s11032-009r-r9327-2

21 Yang, S. et al. (2006) Low level of genetic diversity in cultivated pigeonpea compared to its wild relatives is revealed by diversity arrays technology. Theor. Appl. Genet. 113, 585-595

22 Wenzl, P. et al. (2004) Diversity arrays technology (DArT) for wholegenome profiling of barley. Proc. Natl. Acad. Sci. USA 101, 9915-9920

23 Mace, E.S. et al. (2008) DArT markers, diversity analyses and mapping in Sorghum bicolor. BMC Genomics 9, 26

24 Hippolyte, I. et al. (2010) A saturated SSR/DArT linkage map of Musa acuminata addressing genome rearrangement among bananas. $B M C$ Plant Biol. 10, 65

25 Grewal, T.S. et al. (2008) Mapping quantitative trait loci associated with barley net blotch resistance. Theor. Appl. Genet. 116, 529-539

26 Matsumoto, T. et al. (2008) Development in rice genome research based on accurate genome sequence. Int. J. Plant Genomics, Article ID 348621

27 Cone, K.C. et al. (2002) Genetic, physical, and informatics resources for maize. On the road to an integrated map. Plant Physiol. 130, 15981605

28 Gupta, P.K. et al. (2008) Wheat genomics, present status and future prospects. Int. J. Plant Genomics, Article ID 896451

29 Sreenivasulu, N. et al. (2008) Barley genomics, an overview. Int. J. Plant Genomics, Article ID 486258

30 Paterson, A.H. et al.(2008) Genomics of sorghum. Int. J. Plant Genomics, Article ID 362451

31 Lightfoot, D. (2008) Soybean genomics, developments through the use of cultivar "Forrest". Int. J. Plant Genomics, Article ID, 793158

32 Schlueter, J.A. et al. (2008) BAC-end sequence analysis and a draft physical map of the common bean (Phaseolus vulgaris L.) genome. Trop. Plant Biol. 1, 40-48

33 Bryan, G.J. and Hein, I. (2008) Genomics resources and tools for gene function analysis in potato. Int. J. Plant Genomics, Article ID 216513

34 Luo, M-C. et al. (2003) High-throughput fingerprinting of bacterial artificial chromosomes using the SNAPshot labeling kit and sizing of restriction fragments by capillary electrophoresis. Genomics 82,378 389

35 Ma, Y. et al. (2009) Genomic resources of cowpea (Vigna unguiculata). In Plant and Animal Genome Conference XVII, P393. (www.intlpag.org/17/abstracts/P05f_PAGXVII_393.html)

36 Luo, M.C. et al. (2009) A BAC based fingerprint map of the cassava genome. In Plant and Animal Genome Conference XVII, P158. (www.intl-pag.org/17/abstracts/W21_PAGXVII_158.html)

37 Yuksel, B. and Paterson, A.H. (2005) Construction and characterization of peanut HindIII BAC library. Theor. Appl. Genet. $111,630-639$

38 Guimaraes, P. et al. (2008) BAC libraries construction from the ancestral diploid genomes of the allotetraploid cultivated peanut. BMC Plant Biol. 8, 14
39 Varshney, R.K. et al. (2009) Next-generation sequencing technologies and their implications for crop genetics and breeding. Trends Biotechnol. 27, 522-530

40 Deschamps, S. et al. (2010) Utilization of next-generation sequence platforms in plant genomics and genetic variant discovery. Mol. Breed. $25,553-570$

41 Rounsley, S. et al. (2009) De Novo next generation sequencing of plant genomes. Rice 2, 35-43

42 Huang, S. (2009) The genome of the cucumber, Cucumis sativus L. Nature Genet. 41, 1275-1281

43 Muchero, W. et al. (2009) A consensus genetic map of cowpea [Vigna unguiculata (L) Walp.] and synteny based on EST-derived SNPs. Proc. Natl. Acad. Sci. U. S. A. 106, 18159-21816

44 Varshney, R.K. et al. (2009) A comprehensive resource of drought- and salinity responsive ESTs for gene discovery and marker development in chickpea (Cicer arietinum L). BMC Genomics 10, 523

45 Raju, N.L. et al. (2010) The first set of EST resource for gene discovery and marker development in pigeonpea (Cajanus cajan L.). BMC Plant Biol. 10, 45

46 Rajaram, V. et al. (2010) Development of EST resources in pearl millet and their use in development and mapping of EST-SSRs in four RIL populations. In Plant and Animal Genome Conference XVIII, P. 373. (www.intl-pag.org/18/abstracts/P05d_PAGXVIII_373.html)

47 Schafleitner, R. et al. (2010) 454 pyrosequencing of sweetpotato (Ipomoea batatas) transcriptome. In Plant and Animal Genome Conference XVIII, P. 23. (www.intl-pag.org/18/abstracts/P01_ PAGXVIII_023.html)

48 McNally, K.L. et al. (2009) Genome wide SNP variation reveals relationships among landraces and modern varieties of rice. Proc. Natl. Acad. Sci. U. S. A. 106, 12273-12278

49 Yan, J. et al. (2009) Genetic characterization and linkage disequilibrium estimation of a global maize collection using SNP markers. PLoS ONE 4, e8451

50 Gore, M.A. et al. (2009) A first-generation haplotype map of maize. Science 326, 1115-1117

51 Akhunov, E. et al. (2009) Single nucleotide polymorphism genotyping in polyploid wheat with the Illumina Golden Gate assay. Theor. Appl. Genet. 119, 507-517

52 Close, T.J. et al. (2009) Development and implementation of highthroughput SNP genotyping in barley. BMC Genomics 10, 582

53 Hyten, D.L. et al. (2008) High-throughput genotyping with the GoldenGate assay in the complex genome of soybean. Theor. Appl. Genet. 116, 945-952

54 Penmetsa, R.V. et al. (2010) Studies of genome evolution in papilionoid legumes. In Plant and Animal Genome Conference XVIII, W178. (www.intl-pag.org/18/abstracts/W24_PAGXVIII_178.html)

55 Ribaut, J.M. et al. (2010) Molecular breeding in developing countries, challenges and perspectives. Curr. Opin. Plant Biol. 13, 213-218

56 Goff, S.A. et al. (2002) A draft sequence of the rice genome (Oryza sativa L. ssp. japonica). Science 296, 92-100

$57 \mathrm{Yu}$, J. et al. (2002) A draft sequence of the rice genome (Oryza sativa L. ssp. indica). Science 296, 79-92

58 International Rice Genome Sequencing Project (2005) The map-based sequence of the rice genome. Nature 436, 793-800

59 Paterson, A.H. et al. (2009) The Sorghum bicolor genome and the diversification of grasses. Nature 457, 551-556

60 Schnable, P.S. et al. (2009) The B73 maize genome, complexity, diversity, and dynamics. Science $326,1112-1115$

61 Paux, E. et al. (2008) A physical map of the 1-gigabase bread wheat chromosome 3B. Science 322, 101-104

62 Mayer, K.F.X. et al. (2009) Gene content and virtual gene order of barley chromosome 1H. Plant Physiol. 151, 496-505

63 Timko, M.P. et al. (2008) Sequencing and analysis of the gene-rich space of cowpea. BMC Genomics 9, 103

64 Brown, A.H.D. (1989) Core collection, a practical approach to genetic resources management. Genome 31, 818-824

65 The CGIAR Generation Challenge Programme at 6 (2010) From discovery to application and impact in crop improvement. Brochure of Generation Challenge Programme, Mexico. (www.generationcp.org/ UserFiles2/File/Research/Transit-strategy/Impacts-Phase-I-GCP.pdf)

66 Upadhyaya, H.D. et al. (2008) Genetic structure, diversity, and allelic richness in composite collection and reference set in chickpea (Cicer arietinum L.). BMC Plant Biol. 8, 106-138 
67 Gutierrez, A.G. et al. (2010) Identification of a Rice stripe necrosis virus resistance locus and yield component QTLs using Oryza sativa $\times O$. glaberrima introgression lines. BMC Plant Biol. 10, 6

68 Fonceka, D. et al. (2009) Genetic mapping of wild introgressions into cultivated peanut, a way toward enlarging the genetic basis of a recent allotetraploid. BMC Plant Biol. 9, 103

69 Ribaut, J-M. and Hoisington, D.A. (1998) Marker-assisted selection, new tools and strategies. Trends Plant Sci. 3, 236-239

70 Moretzsohn, M.C. et al. (2009) A linkage map for the B-genome of Arachis (Fabaceae) and its synteny to the A-genome. BMC Plant Biol. 9,40

71 Varshney, R.K. et al. (2009) The first SSR-based genetic linkage map for cultivated groundnut (Arachis hypogaea L.). Theor. Appl. Genet. 118, 729-739

72 Magalhaes, J.V. et al. (2007) A gene in the multidrug and toxic compound extrusion (MATE) family confers aluminum tolerance in sorghum. Nature Genet. 39, 1156-1161
73 Okogbenin, E. et al. (2007) Marker-assisted introgression of resistance to cassava mosaic disease into latin American germplasm for the genetic improvement of cassava in Africa. Crop. Sci. 47, 1895-1904

74 Hash, C.T. et al. (2006) Teamwork delivers biotechnology products to Indian small-holder crop-livestock producers, pearl millet hybrid "HHB 67 improved" enters seed delivery pipeline. SAT eJournal, ejournal.icrisat.org, $2, \mathrm{p} 3$

75 William, H.M. et al. (2007) Wheat breeding assisted by markers, CIMMYT's experience. Euphytica 157, 307-319

76 Thomson, M.J.et al. (2010) Marker assisted breeding. In Abiotic Stress Adaptation in Plants: Physiological, Molecular and Genomic Foundation (Pareek, A. et al., eds), pp. 451-469, Springer

77 Septiningsih, E.M. et al. (2009) Development of submergence-tolerant rice cultivars: the Sub1 locus and beyond. Ann. Bot. 103, 151-160

78 Alpuerto, V.E.B. et al. (2009) Economic impact analysis of markerassisted breeding for tolerance to salinity and phosphorous deficiency in rice. Rev. Agr. Econ. 31, 779-792 